\title{
25 Research Soure \\ CRISPR / Cas-9 Vector System: Targets UL-39 and Inhibits HSV-1 Replication in Vitro
}

Jéssica Vasques Raposo

Instituto Oswaldo Cruz

Luciana Rodrigues Carvalho Barros

INCA: Instituto Nacional de Cancer

Lauana Ribas Torres

IOC: Instituto Oswaldo Cruz

Rafaela Barbosa da Silva Pinto

IOC: Instituto Oswaldo Cruz

Amanda de Oliveira Lopes

IOC: Instituto Oswaldo Cruz

Elen Mello de Souza

IOC: Instituto Oswaldo Cruz

Martin Hernan Bonamino

INCA: Instituto Nacional de Cancer

Vanessa Salete de Paula ( $\nabla$ vdepaula.fiocruz@gmail.com )

Fundação Oswaldo Cruz https://orcid.org/0000-0002-6314-754X

\section{Research Article}

Keywords: HSV-1, sgRNA, Fluorescence, CRISPR/Cas9

Posted Date: October 27th, 2021

DOI: https://doi.org/10.21203/rs.3.rs-833312/v1

License: (c) (1) This work is licensed under a Creative Commons Attribution 4.0 International License.

Read Full License 


\section{Abstract}

HSV-1 affects approximately $67 \%$ of the world population. Here, we sought to use the CRISPR / Cas 9 system with the UL39 target, essential for virus replication. The sgRNA sequence was inserted into plasmid (PX459-UL39). Vero cells were transfected with PX459-UL39, and inhibition of viral replication was assessed 24 and 48 hours later using plaque assays and fluorescence and qPCR. Fluorescence analyzes revealed the presence of anti-HSV-1 CRISPR/Cas9 within Vero cells, and qPCR showed that the viral load decreased by $>95 \%$ of cells transfected with anti-HSV-1 CRISPR / Cas9. Our data demonstrate the usefulness of the PX459-UL39 to inhibit HSV-1 infection.

\section{Main Text}

Human alphaherpesvirus 1 (HSV-1) infection occurs via small lesions in the epithelium, especially around mucous membranes. Once inside the body, the virus establishes a productive infection that results in a localized lesion [1]. These can occur around the lips and/or genitals, where they are referred to as cold sores. HSV-1 produces an acute primary infection ( $90 \%$ of the time asymptomatic), followed by the establishment of latent infection in sensory ganglia, with periods of recurrence. Clustered Regularly Interspaced Short Palindromic Repeats/CRISPR-associated (CRISPR/Cas), an ancient antiviral system that was discovered in bacteria, has shown tremendous potential as a precise, invariant genome-editing tool [2]. Since it was discovered with a genetic editing technique, the CRISPR/Cas9 mechanism as an antiviral has been used frequently in studies in vitro and in vivo. Some studies have already used this technique to inhibit HBV, EBV, HPV and even HSV-1 [3][4][5] Recent data suggest that HSV-1, even during lytic infection, may be an excellent target for treatment using a CRISPR/Cas-based approach [2]. One of these proteins is ICP6, transcribed from the UL39 gene encoding the larger subunit of the enzyme ribonucleotide reductase, which functions to convert ribonucleotide into deoxyribonucleotides [6]. Here, we constructed a CRISPR/Cas9 anti-HSV-1 complex that targets UL39.

We further showed that it is capable of inhibiting the HSV-1 replication and thus is a potential candidate for herpes therapy. For infection, the medium was removed and Vero cells at $80 \%$ confluence were infected with HSV-1 strain KOS for $1 \mathrm{~h}$ at a multiplicity of infection (MOI) of 0.1 and 0.001 in RPMI medium at $37^{\circ} \mathrm{C}$ in a humidified $5 \% \mathrm{CO}_{2}$ environment. Thereafter, cell cultures were maintained at $37^{\circ} \mathrm{C}$ for 24 and $48 \mathrm{~h}$ in $5 \% \mathrm{CO}_{2}$, and the cytopathic effect was assessed by light microscopy. The culture supernatant was subsequently collected, centrifuged at $250 \times \mathrm{g}$ for $10 \mathrm{~min}$ to remove cellular debris, and stored at $-70^{\circ} \mathrm{C}$. Single guide RNAs (sgRNAs) targeting the sequence of UL39 were designed using the online tool, CRISPOR (www.CRISPOR.org). The genomic region surrounding UL39 targeted by the CRISPR guide RNA, gRNA-17 (gRNA-17-UL39), was amplified using primers synthesized by Integrated DNA Technologies (IDT), as described in Table 1.

Vectors based on PX459 (Addgene, \#62988) containing the sgRNA were constructed. sgRNA, formed by annealing forward and reverse gRNA-17-UL39, was cloned into the Bbsl (Bpil; ThermoFisher Scientific, MA, USA) restriction site in the PX459 plasmid according to the Ran protocol [7]. Cloned plasmids were 
transformed into competent DH5a Escherichia coli, after which single clones were isolated and evaluated by restriction enzyme digestion and Sanger-based sequencing. The 41-bp target UL39 sequence (oligonucleotides pRGS-CR-GFP.

For each sample to be transfected, the DNA $(0.5-5 \mu \mathrm{g} / \mu \mathrm{L})$ was diluted in $50 \mu \mathrm{L}$ of serum-free medium. Lipofectamine 2000 (Thermo Fischer) was also diluted in $50 \mu \mathrm{L}$ of medium and incubated for five minutes at room temperature. Then, the diluted DNA was combined with Lipofectamine 2000 , forming a total volume of $100 \mu \mathrm{L}$. The DNA + Lipofectamine 2000 complex was mixed gently and incubated for twenty minutes. The complex was added to each well that already contained cells and culture medium. To ensure mixing and functioning of the complex, the plate was incubated at a temperature of $37^{\circ} \mathrm{C}$, with the presence of $\mathrm{CO}_{2}$ for 24 hours.

Table 1. Oligonucleotides used in the study.

\begin{tabular}{ll}
\hline \multicolumn{1}{c}{ Name } & \multicolumn{1}{c}{ Sequence } \\
\hline gRNA 17 UL-39 forward & 5' CACCGCGCTTCGACGGGAGAGGATG 3' \\
gRNA 17 UL-39 reverse & 5'AAACCATCCTCTCCCGTCGAAGCGC 3' \\
pRGS-CR-GFP forward & 5' GGACATCACCTCCCACAAC 3' \\
pRGS-CR-GFP reverse & 5' GTTTACGTCGCCGTCCAG 3' \\
Primer HHV-1 UL-39 forward & 5'-CCAACTGCACCATGATCATCGA-3' \\
Primer HHV-1 UL-39 reverse & 5'-GATGTTTGTCACCGCAACGAA-3' \\
HHV-1 UL-39 probe & FAM 5'-CCCGCGCACCACGTC-3' MGB \\
\hline
\end{tabular}

Cell viability was assessed using MTT (3-[4,5-dimethylthiazol-2-Yl]-2,5-diphenyltetrazolium bromide) assays. Uninfected Vero cells were seeded into 96-well plates at $30 \times 10^{3}$ cells/well and 24 hafter plating were incubated with different amounts $(0.1,0.2,0.4$ and $0.6 \mu \mathrm{L} /$ well) of Cas 9 master mix for 24 and $48 \mathrm{~h}$ at $37^{\circ} \mathrm{C}$. Thereafter, cells were incubated with the MTT reagent for $4 \mathrm{~h}$ at $37^{\circ} \mathrm{C}$, and cell viability, expressed as a percentage, was assessed by measuring the color intensity of formaan crystals produced by metabolically viable cells at $570 \mathrm{~nm}$ using a spectrophotometer. Vero cells were plated onto 96-well plates at $10 \times 104$ cells/well in $500 \mu \mathrm{L}$ of growth medium. According to the lipofectamine 2000 transfection kit, after reaching $~ 80 \%$ confluence and density of $1 \times 10^{5}$ cell/well, the cells were infected with HSV-1 $\left(10^{9}\right.$ copies $\left./ \mathrm{mL}\right)$ at MOls of 0.1 and 0.001 for $1 \mathrm{~h}$, and $24 \mathrm{~h}$ later were transfected with pRGDUL39 plasmid $(0.5-5 \mu \mathrm{g} / \mu \mathrm{L})$ using Lipofectamine. Infected/ non-transfected cultures, uninfected/ transfected cultures and uninfected/non-transfected cultures were used as controls. The culture supernatants were collected, and cells were fixed. Inhibition of viral replication was assessed using plaque assays and fluorescence and qPCR analyses.

Inhibition of HSV-1 replication was assessed by directly measuring a 92-bp HSV-1 DNAfragment by quantitative polymerase chain reaction (qPCR), using previously described UL39 gene primers and probetarget (da Silva, et al. 2016); results were quantified by reference to a standard curve (Lima, et al. 2017) qPCR was performed in a total volume of $20 \mu \mathrm{L}$ using the AgPath-ID PCR kit (Life Technologies, Carlsbad, CA, USA). Each reaction consisted of $12.5 \mu \mathrm{L}$ of $1 \mathrm{x}$ buffer (PCR Buffer), $2.5 \mu \mathrm{L}$ of sense primers $(1 \mu \mathrm{M})$, 
$2.5 \mu \mathrm{L}$ of antisense primers $(1 \mu \mathrm{M}), 2 \mu \mathrm{L}(0.4 \mu \mathrm{M})$ of the specific probe (Table 3$)$ and $1 \mu \mathrm{L}$ of $1 \mathrm{x}$ enzyme. In addition to test samples, qPCR was performed on negative controls and known quantities of the 92-bp HSV-1 DNA fragment used to generate the standard curve.

After transfection with the pRGD-UL39 plasmid, variously treated VERO cells plated on coverslips were fixed in $4 \%$ paraformaldehyde. The coverslips were washed three times with $1 \mathrm{X}$ phosphate-buffered saline (PBS) and incubated with $10 \mu \mathrm{g} / \mathrm{mL}$ of 4,6-diamidino-2-phenylindol (DAPI; Sigma-Aldrich, St. Louis, MO, USA) for 5 min to stain cell nuclei. Coverslips were subsequently slide-mounted with $2.5 \% 1,4$ diazabicycles (2,2,2)-octane (DABCO; Sigma-Aldrich) and immediately analyzed using an AxioObserver photomicroscope (Zeiss Inc., New York, NY, USA) equipped with a 40x objective lens.

To determine the effect of CRISPR/Cas9 targeting of UL39 during HSV-1 replication, we infected VERO cells with HSV-1 and subsequently transfected them with pRGD-UL39 plasmid. Specifically, Vero cell cultures were infected with HSV-1 at MOls of 0.1 and 0.001 for $24 \mathrm{~h}$ at $37^{\circ} \mathrm{C}$, and then were transfected with a pRGD-UL39 plasmid containing an out-of-frame eGFP sequence. The ability of the CRISPR-carrying plasmid to edit the DNA sequence of the target UL39 was evaluated by confirming eGFP expression derived from indels, which promote in-frame GFP expression. Because eGFP was originally out of frame, one-third of genetic modifications in PRGD-UL39 would place the eGFP gene in-frame, leading to eGFP expression. The eGFP fluorescencemonitored $24 \mathrm{~h}$ after transfection showed that cells were efficiently transfected and the UL39 target sequence was successfully edited (Figure 1b).

Cytotoxic effects of pRGD-UL39 plasmid on VERO cells were assessed 24 and 48 hpost-transfection (hpt) by MTT assay. The results of MTT cell viability assays revealed negligible decreases in the percentage of viable cells compared with controls at $24 \mathrm{hpt}(0.5 \%)$ and $48 \mathrm{hpt}(2.6 \%)$, indicating that the pRGD-UL39 plasmid is relatively non-toxic. A light microscopy analysis at $48 \mathrm{hpt} \mathrm{showed} \mathrm{that} \mathrm{most} \mathrm{cells} \mathrm{exhibited} \mathrm{no}$ changes in morphology and revealed very few floating dead cells. Viral growth kinetics were determined over a single infection cycle. Viral load at $48 \mathrm{hpi}$, measured by qPCR, was substantially reduced in cells in which UL39 was targeted by our CRISPR construct. In the absence of HSV-1, viral growth was higher in cells infected at an MOI of 0.1 than those infected at an MOI of 0.001 (Figura 1a).

At both MOls, no replication of HSV-1 was detected by qPCR (two replicate experiments) in cells transfected with the pRGD-UL39 plasmid carrying the CRISPR/Cas9 anti-HSV complex. From these data, we conclude that UL39 targeting by our CRISPR-carrying plasmid inhibits HSV-1 replication in vitro. The standard treatment for herpes is antiviral drugs, but the continued use of these compounds can lead to the development of viral resistance, especially in immunocompromised patients. Previous studies [8][9] [10][11][12] have shown that the use of the CRISPR/Cas9 system is a promising approach for investigating the structure and functions of important HSV-1 genes, including in viral replication. Although some CRISPR/cas9 anti-HSV-1 studies have been previously reported, the current understanding of viral proteins and their functions is far from complete, accounting for difficulties in treating diseases caused by herpesvirus and developing effective vaccines against them; these deficiencies also highlight the need for more research in this area [13]. The gRNA component of CRISPR/Cas9 directs Cas 9 to the 
target DNA site containing a complementary sequence, where it promotes double-strand cleavage, thereby potentially preventing the production of viral particles containing the complete genome or cleaving genes that produce proteins essential for viral replication [10][14][15] As previously described, other genetic editing techniques used for HSV-1 have efficiencies of less than $1 \%$, whereas the CRISPR/Cas9-mediated mutagenesis system is more than $50 \%$ efficient [16]. In the current study, we tested the ability of the CRISPR/Cas9 system to suppress HSV-1 infection through inhibition of HSV-1 replication and the viral load decreased in $>95 \%$ of cells transfected with CRISPR/Cas 9 anti-HSV-1. Inhibition of HSV-1 has previously been demonstrated by Roehm et al., who targeted ICP0, an immediate early protein that participates in the induction of HSV infection by activating the expression of viral and cellular genes [12] and Karpov et al. similarly demonstrated HSV-1 inhibition by targeting three genes-UL8, UL29, and UL52-involved in HSV-1 replication during lytic infection [13]. In contrast to these other studies that used CRISPR/Cas9 as a genetic editing technique to block HSV-1 replication [12][13], we chose the ICP6 protein, encoded by the UL39 gene, as our target for editing. This gene was chosen because it encodes the larger subunit of the ribonucleotide reductase enzyme, which functions to convert ribonucleotides into deoxyribonucleotides and thus is directly related to viral replication [6].

Accordingly, inhibition of ICP6 expression is expected to interfere with the replication of HSV-1. A previous study conducted by our research group specifically targeted the HSV-1 UL39 gene using the small interfering RNA (siRNA) technique in BALB/c mice infected with HSV-1 and herpetic encephalitis showed that the inhibition of viral replication in the brain of mice were from 43.6 to $99.9 \%$; it also decreased symptoms of the disease and increased survival of the animals [17]. These results confirm that UL39 is an essential region for HSV-1 replication, and thus is a viable target of CRISPR-based therapies. The choice of the gRNA sequence is a very important determinant of the effectiveness and specificity of CRISPR/Cas9 system activity. In the present study, gRNA sequences were designed using CRISPOR software, which calculates all available scores and allows the most appropriate selection for the specific test [18]. The selected sequences yielded good scores, bound closer to the beginning of the target sequence, 1 off-target was found (0-0-0-1-0) in the position strand 938 FW and MIT specificity of 100. Overall, the cytotoxicity of CRISPR and reporter constructs following transfection was negligible $(0.5 \%$ and $2.6 \%$ at 24 and $48 \mathrm{hpt}$, respectively), a finding in line with previous reports [13] that supports the use of Vero cells for functional assays of HSV-1 productive infections.

The efficiency of transfection was evaluated by detecting plasmid-derived GFP in Vero cells. Cells that were infected with HSV-1 and transfected with our CRISPR construct $24 \mathrm{~h}$ later displayed GFP signals perinuclear, indicating that the CRISPR system could act in these cells, as repórter [19]. CRISPR/Cas9 antiHSV-1 activity was investigated in cells infected with HSV-1, we infected Vero cells with HSV-1 (MOIs, 0.1 and 0.001) and transfected them with the CRISPR system (pRGD-UL39 plasmid) $24 \mathrm{~h}$ later. The qPCR analyzes were performed $24 \mathrm{~h}$ and $48 \mathrm{~h}$ after pRGD-UL39 plasmid transfection and indicated that HSV-1 viral activity at both an $\mathrm{MOI}$ of 0.1 and 0.001 viral load decreased by $>95 \%$ of cells transfected with CRISPR anti-HSV-1 / Cas9. In our study, we also did the transfection before replication, however the decrease in viral load was only $24 \%$ (data not showed). The best results were observed when the transfection was performed $24 \mathrm{~h}$ after the infection, simulating the action of using an antiviral. These 
findings corroborate previous reports that inhibition of UL39 results in impaired HSV-1 replication [17]. Our results also compare favorably with previous reports on the use of the CRISPR/Cas9 system to inhibit HSV-1 replication. In the study that targeted the genes UL8, UL29 and UL52, HSV-1 reproduction was suppressed in $90 \%$ of infected Vero cells [13], whereas the study that targeted the ICP0 protein demonstrated viral suppression in $85-90 \%$ of cells [12]. These findings reinforce the importance of studying different genes involved in HSV-1 replication and underscore the potential of the CRISPR/Cas 9 anti-HSV-1 system to inhibit viral replication.

Our findings showed that a CRISPR/Cas9 system targeting the UL39 gene suppressed HSV-1 reproduction in infected Vero cells. The importance of evaluating the duration of antiviral action is well known. In our study, an evaluation of antiviral action at $2 \mathrm{~d}$ revealed potent viral inhibition. However, it has been previously reported that the anti-HSV-1 activity of CRISPR/Cas 9 can be maintained for at least $6 \mathrm{~d}$ after HSV-1 infection [13]. In summary, our findings demonstrate that, in addition to exhibiting low cell cytotoxicity and localization to the cell perinuclear, our CRISPR Cas9/gRNA editing system targeting the UL39 gene successfully inhibited viral replication $48 \mathrm{~h}$ after infection at different MOls. Thus, the genetic constructs used here could represent a promising approach for future in vivo studies as well as new antiviral drug-development efforts.

\section{Declarations}

Funding: This study was financed by the Conselho Nacional de Desenvolvimento Científico e Tecnológico (CNPq), Coordenação de Aperfeiçoamento de Pessoal de Nível Superior - Brasil (CAPES) Finance Code 001, Fundação de Amparo à Pesquisa do Estado do Rio de Janeiro (FAPERJ) and Oswaldo Cruz Institute who approved the project and funded the research with scholarship and grants.

Acknowledgments: We are very grateful for the assistance provided by Timo's Research Laboratory, especially Vinicius Cotta de Almeida and Arnon Jurberg for the advices in this study.

Conflicts of Interest: No reported conflicts. Conflicts that the editors consider relevant to the content of the manuscript have been disclosed.

\section{References}

1. Petti S, Lodi G (2019);The controversial natural history of oral herpes simplex virus type 1 infection. Oral Dis 25: 1850-1865. doi: 10.1111/odi.13234. Epub 2019 Nov 27. PMID: 31733122.

2. Kennedy EM, Kornepati AVR, Mefferd AL, Marshall JB, Tsai K, Bogerd HP, Cullen BR (2015) Optimization of a multiplex CRISPR/Cas system for use as an antiviral therapeutic. Methods 91:8286. doi: 10.1016/j.ymeth.2015.08.012. Epub 2015 Aug 17. PMID: 26291065; PMCID: PMC4684739

3. Zhen S, Lu J, Hua L, Liu Y, Chen W, Li X (2018) WITHDRAWN: CRISPR/Cas9 mediated HPV and PD1 inhibition produces a synergistic anti-tumor effect on cervical cancer. Arch Biochem Biophy DOI: 10.1016/j.abb.2018.10.004 PMID: 30315769 
4. Li H, Sheng C, Liu H, Wang S, Zhao J, Yang L, Jia L, Li P, Wang L, Xie J, Xu D, Sun Y, S, Qiu S, Song, H (2018) Inhibition of HBV Expression in HBV Transgenic Mice Using AAV-Delivered CRISPR-SaCas9. Front Immunol. DOI: 10.3389/fimmu.2018.02080 PMID: 30254645 PMCID PMC614173

5. Huo H, Hu G (2019) CRISPR/Cas9-mediated LMP1 knockout inhibits Epstein-Barr virus infection and nasopharyngeal carcinoma cell growth. Infect Agent Canc. DOI: 10.1186/s13027-019-0246-5PMID: 31673282 PMCID: PMC681617

6. Mostafa HH, Thompson TW, Konen AJ, Haenchen SD, Hilliard JG, Macdonald SJ, Morrison LA. Davido DJ (2018) Herpes Simplex Virus 1 Mutant with Point Mutations in UL39 Is Impaired for Acute Viral Replication in Mice, Establishment of Latency, and Explant-Induced Reactivation. J Virol 92: 01654-17 doi: 10.1128/JVI.01654-17. PMID: 29321311; PMCID: PMC5972887.

7. Ran FA, Hsu PD, Wright J, Agarwala V, Scott DA, Zhang F (2013) Genome engineering using the CRISPR-Cas9 system. Nat Protoc 8: 2281-2308. doi: 10.1038/nprot.2013.143. Epub 2013 Oct 24. PMID:24157548; PMCID: PMC3969860.

8. Wang J, Quake SR (2014) RNA-guided endonuclease provides a therapeutic strategy to cure latentherpesviridae infection. Proc Natl Acad Sci U S A 111: 13157-62.doi:

10.1073/pnas.1410785111. Epub 2014 Aug 25. PMID: 25157128; PMCID: PMC424930.

9. Lin C, Li H, Hao M, Xiong D, Luo Y, Huang C, Yuan Q, Zhang J, Xia N (2016) Increasing the Efficiency of CRISPR/Cas9-mediated Precise Genome Editing of HSV-1 Virus in Human Cells. Sci Rep 6: 34531.doi: 10.1038/srep34531. PMID: 27713537; PMCID: PMC5054376.

10. van Diemen FR, Kruse EM, Hooykaas MJ, Bruggeling CE, Schürch AC, van Ham PM, Imhof SM,Nijhuis M, Wierd EJ, Lebbink RJ (2016) CRISPR/Cas9-Mediated Genome Editing of Herpesviruses Limits Productive and Latent Infections. PLoS Pathog 12: 1005701 doi:

10.1371/journal.ppat.1005701.PMID: 27362483; PMCID: PMC4928872.

11. Xu S, Schafer X, Munger J (2016) Expression of Oncogenic Alleles Induces Multiple Blocks to Human Cytomegalovirus Infection. J Virol 90: 4346-4356.doi: 10.1128/JVI.00179-16. PMID: 26889030; PMCID:PMC4836323

12. Roehm PC, Shekarabi M, Wollebo HS, Bellizzi A, He L, Salkind J, Khalili K (2016) Inhibition of HSV-1 Replication by Gene Editing Strategy. Sci Rep 6: 23146. doi: 10.1038/srep23146. PMI 27064617; PMCID: PMC4827394.

13. Karpov DS, Karpov VL, Klimova RR, Demidova NA, Kushch AA (2019) A PlasmidExpressedCRISPR/Cas9 System Suppresses Replication of HSV Type I in a Vero Cell Culture. Mol Biol (Mosk 53: 91-100. doi: 10.1134/S0026898419010051. PMID: 30895956.

14. Wang G, Zhao N, Berkhout B, Das AT (2018) CRISPR-Cas based antiviral strategies against HIV-1. Virus Res 244: 321-332. doi: 10.1016/j.virusres.2017.07.020. Epub 2017 Jul 29. PMID: 28760348.

15. Moyo B, Bloom K, Scott T, Ely A, Arbuthnot P (2018) Advances with using CRISPR/Cas-mediated gene editing to treat infections with hepatitis B virus and hepatitis C virus. Virus Res 244: 311-320. doi: 10.1016/j.virusres.2017.01.003. Epub 2017 Jan 10. PMID: 28087399. 
16. Chen YC, Sheng J, Trang P, Liu F (2018) Potential Application of the CRISPR/Cas9 System against Herpesvirus Infections. Viruses 10: 291. doi: 10.3390/v10060291. PMID: 29844277; PMCID:PMC6024784.

17. Da Silva AS, Raposo, JV, Pereira, TC, Pinto, MA, De Paula, VS (2016) Effects of RNA Interference Therapy Against Herpes Simplex Virus Type 1 Encephalitis. Antiviral therapy. DOI: 10.3851/IMP3016 PMID: 26669609

18. Haeussler M, Schönig K, Eckert H, Eschstruth A, Mianné J, Renaud JB, Schneider-Maunoury S Shkumatava A, Teboul L, Kent J, Joly JS, Concordet JP (2016) Evaluation of off-target and ontargetscoring algorithms and integration into the guide RNA selection tool CRISPOR. Genome Biol 17:148doi: 10.1186/s13059-016-1012-2. PMID: 27380939; PMCID: PMC4934014

19. Duda K, Lonowski LA, Kofoed-Nielsen M, Ibarra A, Delay CM, Kang Q, Yang Z, Pruett-Miller SM, Bennett EP, Wandall HH, Davis GD, Hansen SH, Frödin M (2014) High-efficiency genome editing via 2A-coupled co-expression of fluorescent proteins and zinc finger nucleases or CRISPR/Cas9 nickas pairs. Nucleic Acids Res 42: 84. doi: 10.1093/nar/gku251. Epub 2014 Apr 21. PMID: 24753413; PMCID: PMC4041425.

\section{Figures}


Figure 1a
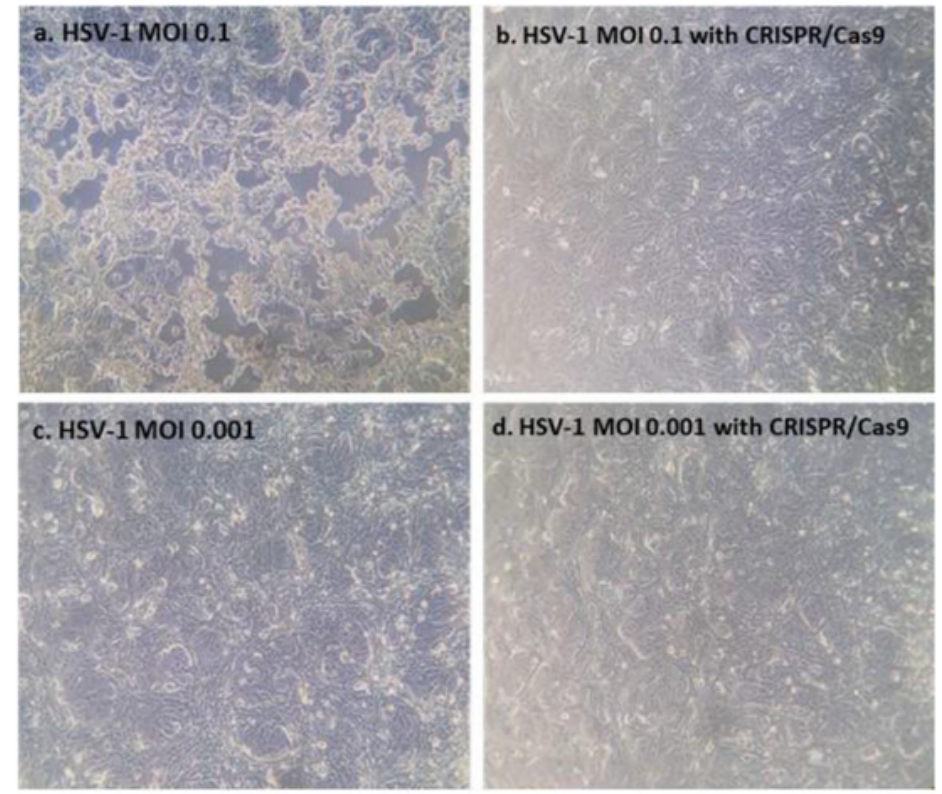

Figure 1b
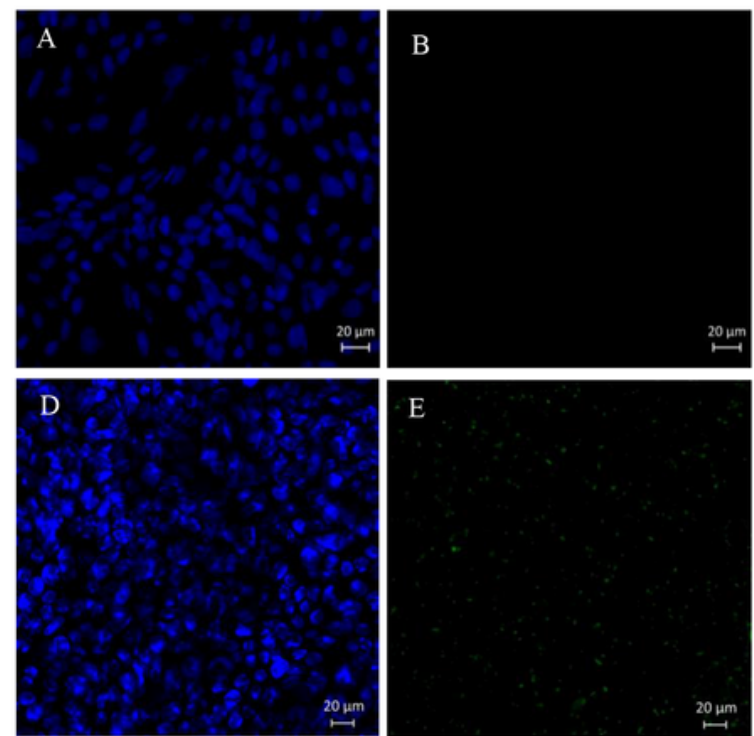

E
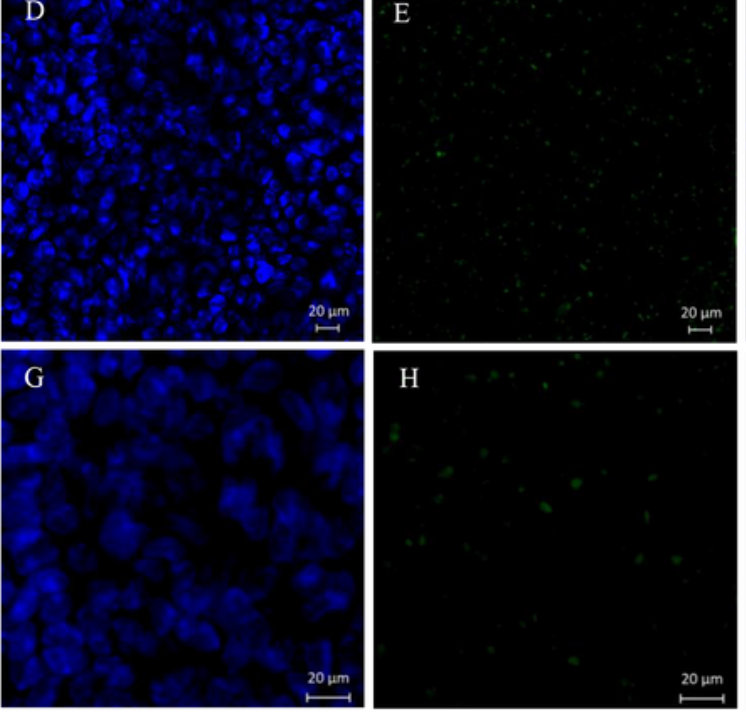

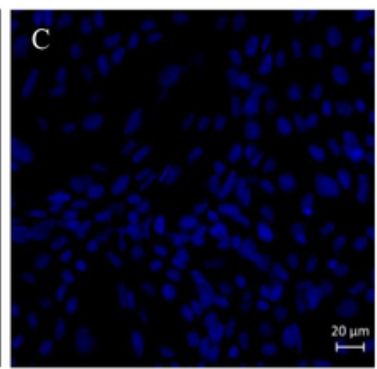

F
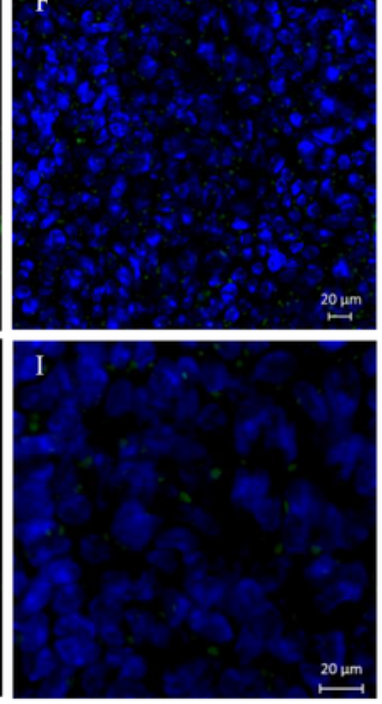

\section{Figure 1}

Figure 1a. Suppression of HSV-1 replication in Vero cells transfected with the pRGD-UL39 plasmid. (a and c) Untreated Vero cell infected with HSV-1. (b and d) Vero cells infected with HSV-1 andtreated with pRGD-UL39. Figure1b. GFP fluorescence in Vero cells. ( $a, b$ and c) Uninfected Vero cells transfected with pRGD-UL39 plasmid, showing DAPI-stained cell nuclei (blue) (a); absence of GFP (green),indicating no shift in the GFP reading frame (b); and merged image (c). (d, e and f) VERO cellstransfected with pRGD- 
UL39 plasmid and infected with HSV-1, showing DAPI-stained cell nuclei blue) (d); presence of green fluorescence, indicative of effective shifting of the GFP reading frame(e); and merged image $(\mathrm{f}) . \mathrm{g}, \mathrm{h}$ and i) Higher-magnification images (magnification, 40x) of panels $d(g)$, e (h) and f (i). 